\title{
creative
commons
}

ISSN 2590-9770

The Art of Discrete and Applied Mathematics 1 (2018) \#P1.07

https://doi.org/10.26493/2590-9770.1244.720

(Also available at http://adam-journal.eu)

\section{Hamiltonicity of token graphs of fan graphs*}

\author{
Luis Manuel Rivera \\ Unidad Académica de Matemáticas, Universidad Autónoma de Zacatecas, \\ Calzada Solidaridad entronque Paseo a la Bufa, Zacatecas, Zac. CP. 98000, México \\ Ana Laura Trujillo-Negrete \\ Departamento de Matemáticas, Cinvestav, \\ Av. IPN \#2508, Col. San Pedro Zacatenco, México, Cd. de México, CP. 07360, México
}

Received 25 July 2017, accepted 8 October 2017, published online 13 February 2018

\begin{abstract}
In this note we show that the token graphs of fan graphs are Hamiltonian. This result provides another proof of the Hamiltonicity of Johnson graphs and also extends previous results obtained by Mirajkar and Priyanka on the token graphs of wheel graphs.
\end{abstract}

Keywords: Token graphs, Johnson graphs, Hamiltonian graphs.

Math. Subj. Class.: 05C38, 05C45

\section{Introduction}

Let $G$ be a simple graph of order $n$ and let $k$ be an integer such that $1 \leq k \leq n-1$. The $k$-token graph, or symmetric kth power, of $G$ is the graph $G^{(k)}$ whose vertices are the $k$-subsets of $V(G)$ and two vertices are adjacent in $G^{(k)}$ if their symmetric difference is an edge of $G$. A classical example is the Johnson graph $J(n, k)$, which is isomorphic to the $k$-token graph of the complete graph $K_{n}$. This class of graphs is widely studied and has connections with coding theory $[7,9,11,13,15]$ (another connection of token graphs with coding theory was showed in [18]).

The definition of $k$-token graphs (without a name) appeared in a work of Rudolph [17], in connection with problems in quantum mechanics and with the graph isomorphism problem. Rudolph presented examples of cospectral graphs $G$ and $H$ such that their corresponding 2-token graphs are not cospectral. Audenaert et al. [3], proved that the 2-token graphs

\footnotetext{
* Part of this work was made when the second author was a master student at Universidad Autónoma de Zacatecas. The authors would like to thank the anonymous reviewer for his/her corrections and suggestions.

E-mail addresses: luismanuel.rivera@gmail.com (Luis Manuel Rivera), 1trujillo@math.cinvestav.mx (Ana Laura Trujillo-Negrete)
}

(a) (i) This work is licensed under http://creativecommons.org/licenses/by/3.0/ 
of strongly regular graphs with the same parameters are cospectral, and suggested that for a given positive integer $k$ there exists infinitely many pairs of non-isomorphic graphs with cospectral $k$-token graphs. This conjecture was proved by Barghi and Ponomarenko [16] and, independently, by Alzaga et al. [2]. Later, Fabila-Monroy et al. [8] reintroduced the $k$-token graphs as part of several models of swapping in the literature [19, 20], and studied some properties of these graphs: connectivity, diameter, cliques, chromatic number, Hamiltonian paths and Cartesian product of token graphs. This line of research was continued by Carballosa et al. [4] who studied regularity and planarity, de Alba et al. [6], who presented some results about independence and matching numbers, and Mirajkar et al. [14], who studied some covering properties of token graphs. Finally, Leaños and Trujillo-Negrete [12] proved a conjecture of Fabila-Monroy et al. [8] about the connectivity of token graphs and de Alba et al. [5] classified the triangular graphs (in other words, the 2-token graphs of complete graphs) that are Cohen-Macaulay.

A graph is Hamiltonian if it contains a Hamiltonian cycle. It is well known that $J(n, k)$ is Hamiltonian [10, 21], in fact, it is Hamiltonian connected [1]. As was noted in [8], the existence of a Hamiltonian cycle in $G$ does not imply that $G^{(k)}$ contains a Hamiltonian cycle. For example, if $k$ is even then $K_{m, m}^{(k)}$ is not Hamiltonian.

We are interested in graphs $G$ such that its token graphs are Hamiltonian. The fan graph $F_{n}$ is the join of graphs $K_{1}$ and $P_{n-1}$. In this note we show that the token graphs of fan graphs are Hamiltonian. Our result provides another proof that $J(n, k)$ is Hamiltonian, and also extends some of the results obtained by Mirajkar and Priyanka Y. B [14] about the Hamiltonicity of the token graphs of wheel graphs.

\section{Main result}

First we present some definitions and notations. For vertices $u, v$ in graph $G$ we write $u \sim v$ to mean that $u$ and $v$ are adjacent vertices in $G$. We write $G \simeq G^{\prime}$ if $G$ and $G^{\prime}$ are isomorphic graphs. A spanning subgraph of $G$ is a subgraph $H$ such that $V(H)=V(G)$. The following proposition is obvious.

Proposition 2.1. If $H$ is a spanning subgraph of $G$ and $H$ is Hamiltonian then $G$ is Hamiltonian.

One of the main properties of token graphs is that $G^{(1)}$ and $G$ are isomorphic. Moreover, $G^{(k)} \simeq G^{(n-k)}$ for any $k \in\{1, \ldots, n-1\}$. Another known property of token graphs is the following.

Proposition 2.2. If $H$ is a subgraph of $G$ then $H^{(k)}$ is a subgraph of $G^{(k)}$. Even more, if $H$ is a spanning subgraph of $G$ then $H^{(k)}$ is a spanning subgraph of $G^{(k)}$.

For a fan graph $F_{n}$ we assume that the vertices of $P_{n-1}$ are $\{1, \ldots, n-1\}$ and the vertex in $K_{1}$ is labeled as $n$. For vertex $A=\left\{a_{1}, \ldots, a_{k}\right\}$ of $F_{n}^{(k)}$ we use the convention that $a_{1}<\cdots<a_{k}$.

The main result of this note is the following.

Theorem 2.3. Let $n$ and $k$ be positive integers with $n \geq 3$ and $1 \leq k \leq n-1$. Then $F_{n}^{\{k\}}$ is Hamiltonian.

Proof. For $k=1, F_{n}^{(1)} \simeq F_{n}$ which is Hamiltonian so in the rest of the proof we assume that $k \geq 2$. We will show that $F_{n}^{(k)}$ has a Hamiltonian cycle such that the vertices 
$\{n-k, n-k+1, \ldots, n-2, n\}$ and $\{n-k, n-k+1, \ldots, n-2, n-1\}$ are adjacent in the cycle. The sequence of vertices $\{1,3\}\{1,2\}\{2,3\}\{1,3\}$ is a Hamiltonian cycle in $F_{3}^{(2)}$. The proof for $n \geq 4$ is by induction on $k$. First we show the case $k=2$ and $n \geq 4$. The sequence of vertices

$$
\begin{aligned}
& \{1, n-1\}\{1, n\}\{1, n-2\}\{1, n-3\} \ldots\{1,3\}\{1,2\} \\
& \{2, n\}\{2, n-1\}\{2, n-2\}\{2, n-3\} \ldots\{2,4\}\{2,3\} \\
& \quad \vdots \\
& \{n-3, n\}\{n-3, n-1\}\{n-3, n-2\} \\
& \{n-2, n\}\{n-2, n-1\} \\
& \{n-1, n\} \\
& \{1, n-1\}
\end{aligned}
$$

is a Hamiltonian cycle in $F_{n}^{(2)}$, where vertices $\{n-2, n-1\}$ and $\{n-2, n\}$ are adjacent in the cycle. We assume as induction hypothesis that $F_{n^{\prime}}^{\left(k^{\prime}\right)}$ satisfies the conditions whenever $k^{\prime}<k$ and $n^{\prime}>k^{\prime}$.

Claim. Let $S_{i}$ be the subgraph of $F_{n}^{(k)}$ induced by the vertex set

$$
V_{i}=\left\{\left\{a_{1}, \ldots, a_{k}\right\} \in V\left(F_{n}^{(k)}\right): a_{1}=i\right\},
$$

with $1 \leq i \leq n-k$. Then $S_{i} \simeq F_{n-i}^{(k-1)}$.

Proof of Claim. Suppose that $V\left(F_{n-i}\right)=\{i+1, \ldots, n\}$ with $V\left(P_{n-i-1}\right)=\{i+1, \ldots$, $n-1\}$ and $n$ the vertex of $K_{1}$. Then the function $A \mapsto A \backslash\{i\}$ is a graph isomorphism between $S_{i}$ and $F_{n-i}^{(k-1)}$.

We identify $S_{i}$ with $F_{n-i}^{(k-1)}$ using the isomorphism given in the proof of the claim. By induction there exists a Hamiltonian cycle $C_{i}$ in $S_{i}$, where vertices $X_{i}:=\{i, n-$ $k+1, \ldots, n-2, n-1\}$ and $Y_{i}:=\{i, n-k+1, \ldots, n-2, n\}$ are adjacent in $C_{i}$, for $1 \leq i \leq n-k$. Let $P_{i}$ be the Hamiltonian subpath of $C_{i}$ from $X_{i}$ to $Y_{i}$, for $1 \leq i \leq n-k$. Let $Z$ denote the vertex $\{n-k+1, n-k+2, \ldots, n-1, n\}$. Therefore $V_{n-k+1}=\{Z\}$.

Let $D_{i}=\{n-k, n-k+1, \ldots, n-1, n\} \backslash\{i\}$, with $n-k+1 \leq i \leq n$. Then the vertex set $V_{n-k}$ of $S_{n-k}$ is $\left\{D_{n}, D_{n-1}, \ldots, D_{n-k+1}\right\}$. Also, we have $X_{n-k}=D_{n}$ and $Y_{n-k}=D_{n-1}$. Let

$$
Q=D_{n-2} D_{n-3} \ldots D_{n-k+2} D_{n-k+1},
$$

which, in fact, is a path in $S_{n-k}$ because $D_{i} \triangle D_{i-1}=\{i-1, i\}$, for $n-k+2 \leq i \leq n-2$. Now,

$$
\begin{aligned}
X_{n-k} \triangle D_{n-2} & =\{n-2, n\} \\
Y_{n-k} \triangle D_{n-2} & =\{n-2, n-1\} \\
Z \triangle D_{n-k+1} & =\{n-k, n-k+1\}
\end{aligned}
$$


and hence

$$
\begin{aligned}
& X_{n-k} \sim D_{n-2}, \\
& Y_{n-k} \sim D_{n-2}, \\
& D_{n-k+1} \sim Z,
\end{aligned}
$$

in $F_{n}^{(k)}$. Notice that $X_{i} \triangle X_{i+1}=\{i, i+1\}$ and $Y_{i} \triangle Y_{i+1}=\{i, i+1\}$, for $1 \leq i \leq n-k-1$, and $X_{1} \triangle Z=\{1, n\}$. Therefore we can define a Hamiltonian cycle $\mathcal{C}$ in $F_{n}^{(k)}$ as

$$
\begin{aligned}
X_{1} \underset{P_{1}}{\longrightarrow} Y_{1} Y_{2} \underset{P_{2}}{\longrightarrow} X_{2} \ldots X_{(n-k-1)} \underset{P_{n-k-1}}{\longrightarrow} Y_{(n-k-1)} Y_{(n-k)} X_{(n-k)} D_{n-2} \\
\underset{Q}{\longrightarrow} D_{n-k+1} Z X_{1},
\end{aligned}
$$

if $n-k$ is even, and

$$
\begin{aligned}
X_{1} \underset{P_{1}}{\longrightarrow} Y_{1} Y_{2} \underset{P_{2}}{\longrightarrow} X_{2} \ldots Y_{(n-k-1)} \underset{P_{n-k-1}}{\longrightarrow} X_{(n-k-1)} X_{(n-k)} Y_{(n-k)} D_{n-2} \\
\stackrel{\vec{Q}}{\longrightarrow} D_{n-k+1} Z X_{1},
\end{aligned}
$$

if $n-k$ is odd. Furthermore

$\{n-k, n-k+1, \ldots, n-2, n-1\}=X_{n-k} \sim Y_{n-k}=\{n-k, n-k+1, \ldots, n-2, n\}$,

in $\mathcal{C}$, as desired.

The wheel graph $W_{n}$ is the joint graph of $K_{1}$ and $C_{n-1}$. It is known that Johnson graphs [10,21] and the $k$-token graphs of wheel graphs [14] are Hamiltonian, the following corollary provides another proof of this facts.

Corollary 2.4. If $F_{n}$ is a spanning subgraph of $G$ then $G^{(k)}$ is Hamiltonian. In particular the Johnson graphs and the k-token graphs of wheel graphs are Hamiltonian.

Proof. As $F_{n}$ is a spanning subgraph of $G$ then $F_{n}^{(k)}$ is a spanning subgraph of $G^{(k)}$ by Proposition 2.2. The $k$-token graph of $F_{n}$ is Hamiltonian by Theorem 2.3 and hence $G^{(k)}$ is Hamiltonian by Proposition 2.1. In particular $F_{n}$ is a spanning subgraph of $W_{n}$ and $K_{n}$.

\section{References}

[1] B. Alspach, Johnson graphs are Hamilton-connected, Ars Math. Contemp. 6 (2013), 21-23, http://amc-journal.eu/index.php/amc/article/view/291.

[2] A. Alzaga, R. Iglesias and R. Pignol, Spectra of symmetric powers of graphs and the WeisfeilerLehman refinements, J. Comb. Theory Ser. B 100 (2010), 671-682, doi:10.1016/j.jctb.2010.07. 001.

[3] K. Audenaert, C. Godsil, G. Royle and T. Rudolph, Symmetric squares of graphs, J. Comb. Theory Ser. B 97 (2007), 74-90, doi:10.1016/j.jctb.2006.04.002.

[4] W. Carballosa, R. Fabila-Monroy, J. Leaños and L. M. Rivera, Regularity and planarity of token graphs, Discuss. Math. Graph Theory 37 (2017), 573-586, doi:10.7151/dmgt.1959.

[5] H. de Alba, W. Carballosa, D. Duarte and L. M. Rivera, Cohen-Macaulayness of triangular graphs, Bull. Math. Soc. Sci. Math. Roumanie 60 (2017), 103-112, http://s.smr.ro/ bulletin/volumes/60-2/node2.html. 
[6] H. de Alba, W. Carballosa, J. Leaños and L. M. Rivera, Independence and matching numbers of some token graphs, 2016, arXiv:1606.06370 [math.CO].

[7] T. Etzion and S. Bitan, On the chromatic number, colorings, and codes of the Johnson graph, Discrete Appl. Math. 70 (1996), 163-175, doi:10.1016/0166-218x(96)00104-7.

[8] R. Fabila-Monroy, D. Flores-Peñaloza, C. Huemer, F. Hurtado, J. Urrutia and D. R. Wood, Token graphs, Graphs Combin. 28 (2012), 365-380, doi:10.1007/s00373-011-1055-9.

[9] R. L. Graham and N. J. A. Sloane, Lower bounds for constant weight codes, IEEE Trans. Inf. Theory 26 (1980), 37-43, doi:10.1109/tit.1980.1056141.

[10] H. R. Ho, Hamiltonicity of the graph $G(n, k)$ of the Johnson scheme, Jurnal Informatika 3 (2007), 41-47, http://majour.maranatha.edu/index.php/ jurnal-informatika/article/view/263.

[11] S. M. Johnson, A new upper bound for error-correcting codes, IRE Trans. Inf. Theory 8 (1962), 203-207, doi:10.1109/tit.1962.1057714.

[12] J. Leaños and A. L. Trujillo-Negrete, The connectivity of token graphs, submitted.

[13] R. A. Liebler and C. E. Praeger, Neighbour-transitive codes in Johnson graphs, Des. Codes Cryptogr. 73 (2014), 1-25, doi:10.1007/s10623-014-9982-0.

[14] K. G. Mirajkar and P. Y. B, Traversability and covering invariants of token graphs, International J. Math. Combin. 10 (2016), 132-138, http: / / fs . gal lup. unm. edu / I JMC-2-2016. pdf.

[15] M. Neunhöffer and C. E. Praeger, Sporadic neighbour-transitive codes in Johnson graphs, Des. Codes Cryptogr. 72 (2014), 141-152, doi:10.1007/s10623-013-9853-0.

[16] A. Rahnamai Barghi and I. Ponomarenko, Non-isomorphic graphs with cospectral symmetric powers, Electron. J. Comb. 16 (2009), \#R120, http: / / www . combinatorics.org/ Volume_16/Abstracts/v16i1r120.html.

[17] T. Rudolph, Constructing physically intuitive graph invariants, 2002, arXiv:quant-ph/0206068.

[18] N. J. A. Sloane, Sequence A085680 in The On-Line Encyclopedia of Integer Sequences, published electronically at https: / / oeis.org.

[19] J. van den Heuvel, The complexity of change, in: S. R. Blackburn, S. Gerke and M. Wildon (eds.), Surveys in Combinatorics 2013, Cambridge University Press, Cambridge, volume 409 of London Mathematical Society Lecture Note Series, pp. 127-160, 2013, doi:10.1017/ cbo9781139506748.005.

[20] K. Yamanaka, E. D. Demaine, T. Ito, J. Kawahara, M. Kiyomi, Y. Okamoto, T. Saitoh, A. Suzuki, K. Uchizawa and T. Uno, Swapping labeled tokens on graphs, in: A. Ferro, F. Luccio and P. Widmayer (eds.), Fun with Algorithms, Springer, Cham, Switzerland, volume 8496 of Lecture Notes in Computer Science, pp. 364-375, 2014, doi:10.1007/978-3-319-07890-8_31.

[21] F. Zhang, G. Lin and R. Cheng, Some distance properties of the graph $G(n, k)$ of Johnson scheme, 1997, talk presented at the International Congress in Algebras and Combinatorics (Hong Kong, 19-23 August 1997), https://www.math.cuhk.edu.hk/ conference/icac1997/. 